\title{
Silencing of FTX suppresses pancreatic cancer cell proliferation and invasion by upregulating miR-513b-5p
}

\author{
Shan $\mathrm{Li}^{\dagger}$, Qian Zhang ${ }^{\dagger}$, Wen Liu and Chunbo Zhao*
}

\begin{abstract}
Background: Abnormal expression of long non-coding RNA (IncRNA) FTX (five prime to Xist), which is involved in $X$ chromosome inactivation, has been reported in various tumors. However, the effect of FTX on the development of pancreatic cancer (PC) has not been elucidated. The purpose of this study was to explore the possible molecular mechanism of FTX in PC.

Methods: Quantitative real-time PCR (qRT-PCR) was used to measure the expression levels of FTX and miR-513b-5p in PC cell lines. Proliferation and apoptosis of PC cells were determined by CCK-8, Edu assay, and flow cytometry. Invasion and migration ability of PC cells were detected by Transwell assay and scratch test. Bioinformatics analysis, luciferase reporter gene assay, and RNA immunoprecipitation (RIP) assay were used to verify the direct binding between FTX and miR-513b-5p. The xenotransplantation mouse model was established to explore the effect of FTX and miR-513b-5p on the PC tumor growth in vivo.

Results: The expression levels of FTX were increased in PC cell lines, and silencing of FTX remarkably suppressed the invasion ability and cell viability. Besides, FTX could bind to miR-513b-5p as a competitive endogenous RNA, thus promoting the invasion and proliferation ability of PC cells. Moreover, knockdown of FTX inhibited the tumor growth and increased the expression levels of miR-513b-5p and apoptosis-related proteins in vivo.
\end{abstract}

Conclusions: FTX could directly combine with miR-513b-5p as a competitive endogenous RNA, thus promoting the occurrence and development of PC in vitro and in vivo.

Keywords: Pancreatic cancer, Long non-coding RNA FTX, miR-513b-5p, Xenotransplantation mouse model

\section{Background}

Pancreatic cancer (PC) is a malignancy of the digestive tract, which is characterized by high mortality rate, poor prognosis, and extremely low cure rate [1-4]. Currently, the main treatment for $\mathrm{PC}$ is traditional surgery, radiotherapy and chemotherapy [5]. When clinical symptoms appear, it is often in the middle or late stage, and the 5year survival rate of patients is less than $5 \%$ [6]. Invasion

\footnotetext{
* Correspondence: bgfauvqvfd7@163.com

†Shan Li and Qian Zhang contributed equally to this work.

Department of Gastrointestinal Radiation Oncology, Cancer Hospital of Harbin Medical University, 150 Haping Road, Nangang District, Harbin City, Heilongjiang Province 150081, P. R. China
}

and metastasis are important biological characteristics of $\mathrm{PC}$ and the main reasons for poor prognosis of $\mathrm{PC}$ patients [7]. However, due to the inconspicuous early symptoms and the lack of effective biomarkers for early diagnosis, most PC patients have shown local invasion or even distant metastasis when seeking treatment, thus missing the best time for treatment $[8,9]$. Extensive studies have identified various genes and proteins involved in the regulation of PC invasion and metastasis $[10,11]$, and the expression and activation of these genes and proteins are regulated by a variety of molecular pathways [12]. However, the pathogenesis of PC remains

(c) The Author(s). 2021 Open Access This article is licensed under a Creative Commons Attribution 4.0 International License, which permits use, sharing, adaptation, distribution and reproduction in any medium or format, as long as you give appropriate credit to the original author(s) and the source, provide a link to the Creative Commons licence, and indicate if changes were made. The images or other third party material in this article are included in the article's Creative Commons licence, unless indicated otherwise in a credit line to the material. If material is not included in the article's Creative Commons licence and your intended use is not permitted by statutory regulation or exceeds the permitted use, you will need to obtain permission directly from the copyright holder. To view a copy of this licence, visit http://creativecommons.org/licenses/by/4.0/ The Creative Commons Public Domain Dedication waiver (http://creativecommons.org/publicdomain/zero/1.0/) applies to the data made available in this article, unless otherwise stated in a credit line to the data. 
unclear. Therefore, the key to improve the prognosis of PC is to further study the biological characteristics of PC and the molecular regulatory mechanism of its occurrence and development, and to search for reliable markers for early diagnosis and prognosis evaluation.

The development of molecular biology provides new strategies for the early diagnosis and treatment of PC [13-15]. Long non-coding RNAs (lncRNAs) are non-coding RNAs with a length of more than 200 nucleotides and play important regulatory roles in the physiological and pathological processes of the body through epigenetic, transcriptional, and post-transcriptional regulations [16]. LncRNAs are abnormally expressed in various tumor tissues showing tissue specificity, making them ideal diagnostic markers and potential therapeutic targets [17, 18]. Studies have shown that a variety of lncRNAs played important regulatory roles in the invasion and metastasis of PC [19, 20]. For example, HOXA terminal transcriptional antisense RNA (HOTTIP) is highly expressed in pancreatic ductal adenocarcinoma, and silencing of HOTTIP can suppress the growth and metastasis of $\mathrm{PC}$ cells and promote the apoptosis of cancer cells [21]. LncRNA-ROR can trigger the occurrence of epithelial-mesenchymal transition (EMT) and promote the invasion and metastasis of PC [22]. LncRNA-MALAT can maintain the stability of PC stem cells by upregulating SOX2 [19]. Studies have found that lncRNA FTX (five prime to Xist) was abnormally expressed in various cancers, such as hepatocellular carcinoma, renal cell carcinoma and colorectal cancer [23-25], but the regulation of lncRNA FTX in PC remains unclear.

MicroRNAs (miRNAs) are non-coding miRNAs with about 18-25 nucleotide in length and can inhibit the expression of target mRNAs by targeting their 3'-UTR region [26]. Altered expression of miRNAs have been shown to play important roles in the invasion and metastasis of PC [27]. It was reported that miR-513b-5p had low expression levels in gastric cancer, lung cancer and colon cancer, and acted as an oncogene [28-30]. However, the expression of lncRNA FTX in PC and its biological mechanism are unclear.

In this study, the expression levels of IncRNA FTX in PC cell lines were detected, and appropriate PC cells were selected to verify the interaction between lncRNA FTX and miR-513b-5p. In addition, changes in the biological behaviors of PC cells such as proliferation, migration, invasion and apoptosis were detected by inhibiting the expression of IncRNA FTX. The aim of this study was to explore the role of FTX and miR-513b-5p in PC and their possible mechanisms, hoping to provide insights into the early diagnosis and treatment of PC.

\section{Methods}

\section{Cell culture}

HPDE6-C7, HS-766 T, SW1990, AsPC-1P, BxPC-3, and PANC-1 cells were purchased from ATCC (Manassas, VA). RPMI 1640 medium (GIBCO, Carlsbad, CA) supplemented with 10\% Fetal Bovine Serum (FBS, Gibco, Thermo Fisher Scientific) and $1 \%$ streptomycinpenicillin (Gibco, Thermo Fisher Scientific) was used to incubate the cells. All cell lines were cultured in a humidified incubator at $37{ }^{\circ} \mathrm{C}$ with $5 \% \mathrm{CO}_{2}$.

\section{Cell transfection}

Negative control (NC) vector and LV-FTX vector were constructed using lenti-pac HIV expression package mixture and lentiviral vector (Gene Copoeia, Rockville, $\mathrm{Md}$ ) following the manufacturer's instructions. All cell lines were seeded into 6-well plates and incubated overnight to reach $4 \times 10^{5}$ cells per well. The original culture medium was replaced with $2 \mathrm{ml}$ fresh culture medium containing $6 \mathrm{~g} / \mathrm{ml}$ polybrene, and appropriate viral suspension was added for incubation at $37^{\circ} \mathrm{C}$. Transfected cells were incubated for $48 \mathrm{~h}$ for subsequent experiments.

\section{Cell proliferation and apoptosis assay}

Cell viability was determined by CCK-8 kit (Dojindo, Japan). The proliferation capacity was detected using Cell Light $^{\mathrm{tm}}$ EdU kit (RiboBio, Nanjing, China). After pretreatment, $50 \mu \mathrm{M}$ EdU was added to the medium to culture cells for another $2 \mathrm{~h}$. After fixed with 4\% paraformaldehyde, the cells were staining with Hoechst 33342 (1:10,000, Sigma, USA) at room temperature for $10 \mathrm{~min}$. EdU positive cells $\left(1 \times 10^{5}\right)$ were counted by fluorescence microscope (Nikon, Tokyo, Japan). Moreover, cell apoptosis was measured using Annexin VFITC/PI kit (KeyGEN, Nanjing, China) for $15 \mathrm{~min}$ in dark. The Negative control (NC) and LV-FTX cells $(1 \times$ $10^{5}$ ) were washed twice with PBS and re-suspended in PBS, then stained with Annexin V (1:1000) and PI for $15 \mathrm{~min}$ in dark. Apoptosis rates were analyzed using FACSAria flow cytometry (BD Biosciences, Franklin Lakes, NJ).

\section{Cell cycle detection}

Cells were inoculated into a 6-well plate with a cell density of $1 \times 10^{6}$ cells per well. After $24 \mathrm{~h}$, the cells were digested by trypsin and fine cells in the supernatant were collected and washed with PBS for 3 times. Cells were then fixed with $70 \%$ ethanol, and each cell sample was resuspended and added with $500 \mathrm{~L}$ pre-mixed PI dye. Cells were resuspended and incubated in dark for 30 min. GO/G1 cell rations were analyzed using FACSAria flow cytometry (BD Biosciences, Franklin Lakes, NJ). 


\section{Cell migration and invasion assay}

For cell migration assay, all cell lines after transfection were seeded in six-well plates for $48 \mathrm{~h}$ to form a confluent monolayer. Then a $10 \mu \mathrm{l}$ pipette tip was used to scratch cells, and images were captured using a fluorescence microscope (Nikon, Tokyo, Japan) at $0 \mathrm{~h}$ and $24 \mathrm{~h}$ after the wound. ImageJ software (NIH, Bethesda, MD) was used to analyze wound healing to assess cell migration. For cell invasion assay, cell migration was assessed by the number of cells $\left(1 \times 10^{6} / \mathrm{ml}\right)$ passing through a polycarbonate membrane $(8 \mu \mathrm{m}$ well, BD Biosciences, San Jose, CA, USA). In addition, the treated cell suspension was added to the upper chamber of Transwell (Costar, Badhoevedorp, Netherlands) covered with artificial basement membrane, and RPMI 1640 medium containing 10\% Fetal Bovine Serum (FBS, Gibco, Thermo Fisher Scientific) and $1 \%$ streptomycin-penicillin (Gibco, Thermo Fisher Scientific) was added to the lower compartment. Cells were cultured at $37^{\circ} \mathrm{C}$ for $24 \mathrm{~h}$, followed by stained with $5 \%$ crystal violet solution and counted under the fluorescence microscope (Nikon, Tokyo, Japan) to evaluate the invasion ability of the cells.

\section{qRT-PCR and Western blot}

qRT-PCR was performed as described in literature [31]. GAPDH and U6 were used as the internal references. The expression levels of FTX and miR-513b-5p were calculated using $2^{-\Delta \Delta \mathrm{Ct}}$ method. The primer sequences were listed as below:

\section{FTX forward 5' -CAAAGCTGGTCCTGTGCCTG-3'} FTX reverse 5' -ATTGAGTGTGGCATCACCTCC-3' GAPDH forward, 5'-GGGCTGCTTTTAACTCTGGT-3' GAPDH reverse, 5'-GCAGGTTTTTCTAGACGG-3' miR-513b-5p forward; 5' -GGCCGGGGAGCTGGAG AAGA-3 miR-513b-5p reverse; 5' -TCCATGGAGGGTTGGG GGTTCC-3'

U6 forward, 5' -CTCGCTTCGGCAGCACATATATT-3' U6 reverse, 5' -ACGCTTCACGAATTTGCGTGGC-3' .

Western Blot analysis was performed as described in literature [32]. Total proteins were extracted from cells and tissues. BCA method was used to detect the concentration of total proteins. Protein samples were electrophoretic separated by SDS-PAGE gel and transferred to PVDF membrane. Next, BSA was sealed at room temperature for $2 \mathrm{~h}$, and Cyclin D1 (ab16663), PCNA (ab29), c-caspase-3 (ab49822) and c-caspase-12 (ab62463) were incubated at $4{ }^{\circ} \mathrm{C}$ with shaking overnight. Secondary antibodies were then added and incubated at room temperature for $2 \mathrm{~h}$. TBST was cleaned for 3 times. Luminescent solution was used for exposure. GAPDH (ab8245) were used as the internal reference, and the relative expression levels of protein were quantitatively analyzed by ImageJ software.

\section{Luciferase activity and RIP assay}

For detection of luciferase activity, PCR was performed to amplify the partial sequences of FTX which contained the putative binding sites of miR-513b-5p. The sequences were then cloned into the pmirGLO DualLuciferase miRNA Target Expression Vector (Promega Corp., Fitchburg, WI, USA). GeneArt ${ }^{\mathrm{m}}$ Site-Directed Mutagenesis system (Thermo Fisher Scientific, Inc.) was used to induce site-directed mutagenesis of miR-513b$5 \mathrm{p}$ complementary bases in the sequence of FTX. Then, miR-513 mimic or its control was co-transfected with FTX-WT or FTX-MUT, and luciferase activity in the cells was detected using the dual-luciferase Reporter Assay System (Promega, Madison, WI, USA) $24 \mathrm{~h}$ posttransfection. Immunoprecipitation (RIP) was detected using the Magna RIP $^{\text {ma }}$ RNA binding protein immunoprecipitation kit (Millipore, Bedford, MA, USA).

\section{Establishment of mouse xenotransplantation model}

Twelve BALB/c nude mice (female, 8 weeks old, obtained from Shanghai animal experimental center) were used to establish the xenotransplantation model. Briefly, tumor volume was measured every $5 \mathrm{~d}$ after subcutaneous injection of $100 \mu \mathrm{l}$ AsPC- $1\left(1 \times 10^{6}\right.$ cells $)$ suspension (transfected with LV-NC or LV-FTX) into the left abdomen of mice. Mice were anesthetized with $3 \%$ sodium pentobarbital $(30 \mathrm{mg} / \mathrm{kg}$ body weight, Sigma, USA) through intraperitoneal injection and sacrificed by cervical dislocation after $30 \mathrm{~d}$. Animal experiments were conducted in accordance with the Cancer Hospital of Harbin Medical University guidelines for the care and use of experimental animals strictly. All experiments were approved by the Ethics Committee of Cancer Hospital of Harbin Medical University.

\section{Immuno-histochemical staining (IHC) analysis}

The separated PC tumor tissues were embedded in paraffin and sectioned for IHC test. In brief, the tissue sections were incubated with $0.15 \%$ Triton $\mathrm{X}-100$, and then were closed with $1 \%$ goat serum albumin (Flarebio, Wuhan, China). The sections were then incubated with Ki-67 antibody (Abcam, Cambridge, UK) at a dilution of 1:500. Next, the sections were incubated with secondary antibody (1:5000 Abcam, Cambridge, UK) at $37^{\circ} \mathrm{C}$ for $30 \mathrm{~min}$.

\section{Statistical analysis}

All experiments were performed in three independent experiments, and data were expressed as mean \pm standard error (SE). GraphPad Prism 6.0 (GraphPad Software Inc., La Jolla, CA, USA) was used to analyze the data. 
ANOVA and Student's t test were used for significance analysis. $P<0.05$ was considered statistically significant.

\section{Results}

The expression of FTX and miR-513b-5p in PC cell lines

The expressions of FTX and miR-513b-5p in PC cell lines were determined by qRT-PCR. As shown in Fig. 1a, compared with HPDE6-C7 cells, FTX was remarkably upregulated in PC cell lines $(P<0.05)$. Furthermore, compared with the HPDE6-C7 cells, the expression levels of miR-513b-5p in PC cell lines were lower (Fig. 1b, $P<$ 0.05). qRT-PCR results showed that FTX was significantly upregulated, whereas miR-513b-5p had a lower expression levels in PC cells compared with that in HPDE6-C7 cells. These results indicated that FTX and miR-513b-5p might be related to the development of PC.

Effects of silencing of FTX on proliferation, apoptosis and cell cycle of PC cells

To explore the effect of FTX on the biological function of PC cells, LV-FTX was transfected into PANC-1 and SW1990 cells to evaluate the effects of silencing of FTX on the proliferation and apoptosis of PC cells. As shown in Fig. 2a, the expression levels of FTX were markedly decreased in PANC-1 and SW1990 cells transfected with LVFTX $(P<0.05)$, indicating that FTX was successfully silenced by LV-FTX transfection in PC cells. After successful transfection, the effects of silencing of FTX on the cell viability and proliferation ability were assessed. It showed that the cell viability and Edu positive cell number of PANC-1 and SW1990 cells in LV-FTX group were observably reduced compared with that in the negative control group $(P<0.05)$ (Fig. $2 \mathrm{~b}$ and $\mathrm{c}$ ), suggesting that cell viability and proliferation ability were significantly suppressed after silencing of FTX. Furthermore, flow cytometry and western blot were conducted to examine the effect of silencing of FTX on PC cells. The results showed that the apoptosis rates of PANC-1 and SW1990 cells in LV-FTX group were remarkably higher compared to that in the control group (Fig. $2 \mathrm{~d}, P<0.05$ ), indicating that mass apoptosis cells appeared in the LV-FTX transfection group by silencing of FTX. Meanwhile, Western Blot results showed that compared with the control group, the expression levels of cleaved caspase 3 (c-caspase-3) and cleaved caspase 12 (c-caspase-12) were markedly increased in PANC-1 and SW1990 cells of LV-FTX group (Fig. 2e, $P$ $<0.05$ ). As c-caspase- 3 and c-caspase- 12 are family members of caspases that are critical mediators of programmed cell death [33], suggesting that the probability of apoptosis was greatly increased by silencing of FTX. These results demonstrated that silencing of FTX could significantly suppress the proliferation and promote apoptosis of PC cells. Moreover, the ration of G0 and G1 cells was detected by flow cytometry and the expression of Cyclin D1 and PCNA were determined by western blot. It showed that silencing of FTX induced PC cell cycle arrest at G0/G1 phase (Fig. 2d, $P$ $<0.05)$ and decreased the expression levels of Cyclin D1 and PCNA (Fig. 2e, $P<0.05$ ). We therefore concluded that silencing of FTX may inhibit cell proliferation and promote apoptosis by regulating cell cycle.

\section{Effects of silencing of FTX on invasion and migration of PC cells}

In addition, to explore whether silencing of FTX could impact the invasion and migration of PC cells, the migration ability of cells was detected by wound-healing assay, and the results showed that compared with the control group, the migration rates of PANC-1 and SW1990 cells with the silencing of FTX were observably decreased after $24 \mathrm{~h}$ wound (Fig. 3a, $P<0.05$ ). The invasion and migration ability of PC cells were measured by Transwell assay. It showed that the invasion and migration numbers of PANC-1 and SW1990 cells with the silencing of FTX were remarkably decreased compared with the control group (Fig. 3b and c, $P<0.05$ ). These results demonstrated that silencing of FTX suppressed
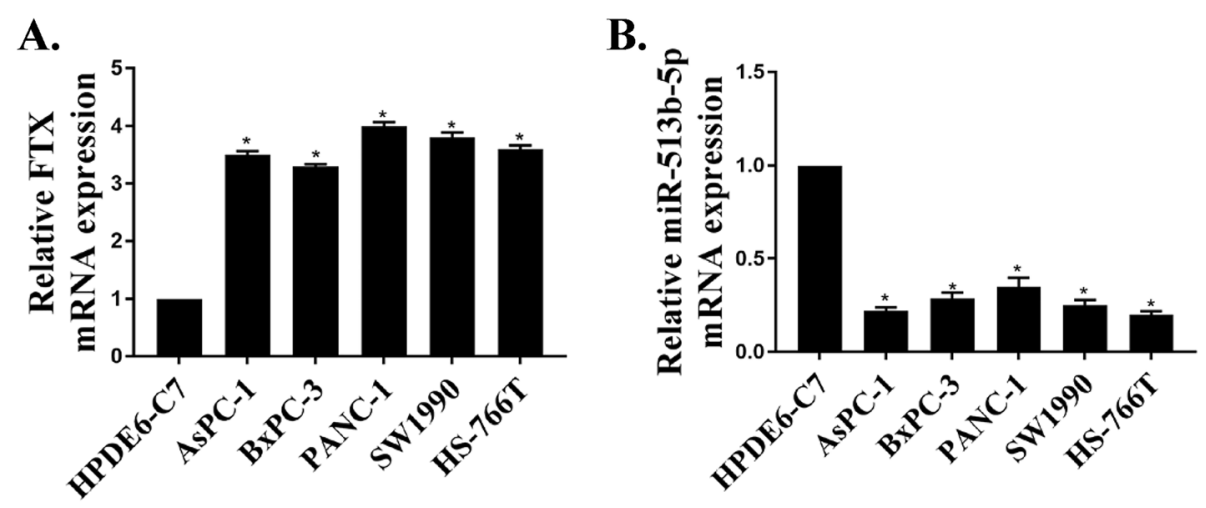

Fig. 1 Expression of FTX and miR-513b-5p in PC cell lines. Detection of the FTX (a) and miR-513b-5p (b) expression in PC cell lines (HPDE6-C7, AsPC-1, BxPC-3, PANC-1, SW1990 and HS-766 T) by qRT-PCR. ${ }^{*} P<0.05$ 

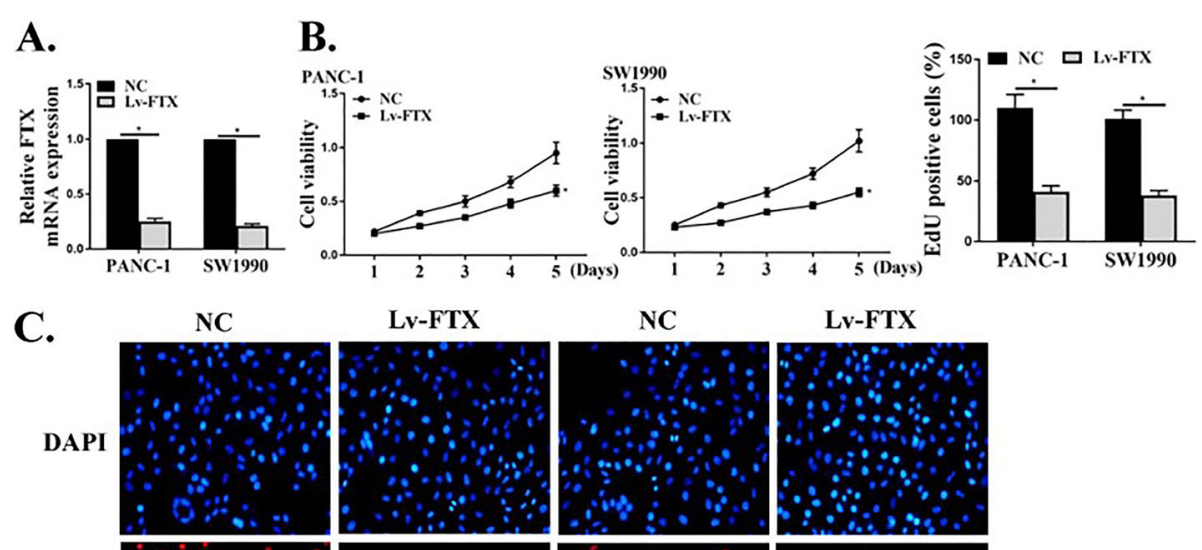

Lv-FTX
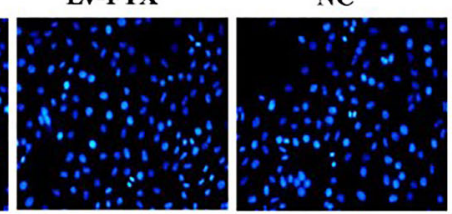

Lv-FTX
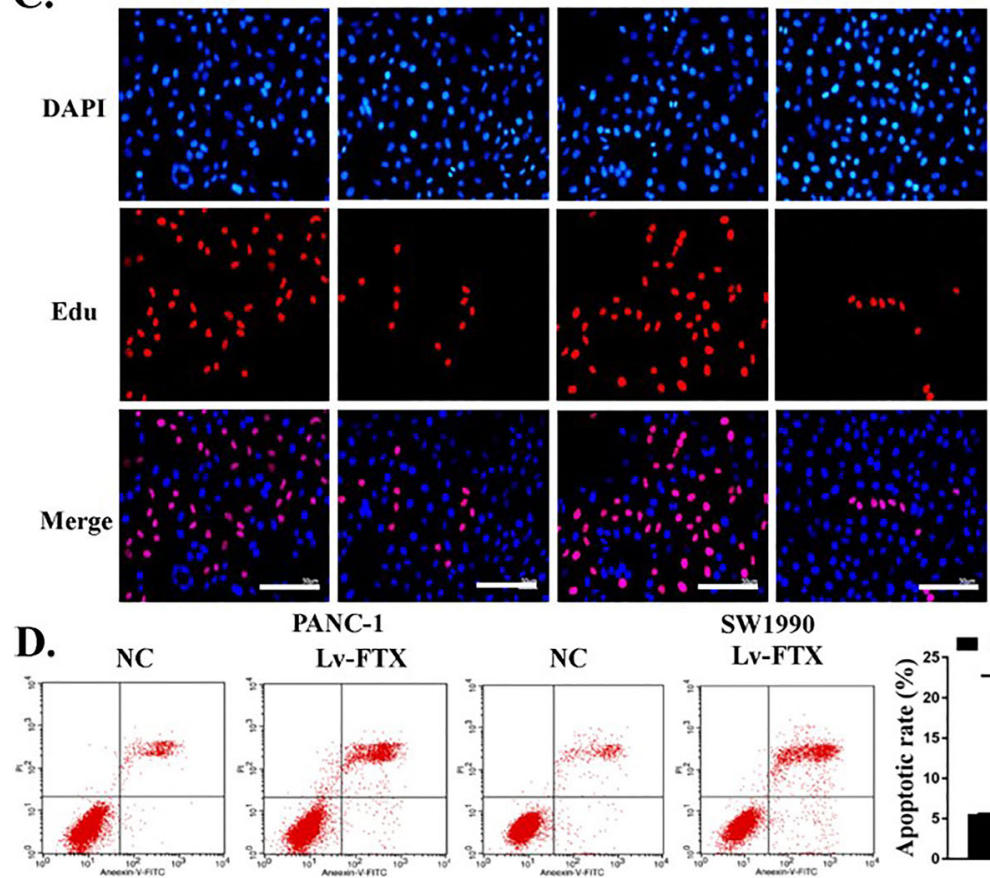

PANC-1

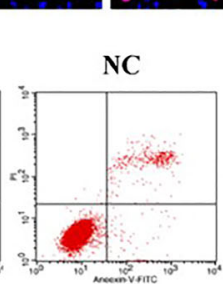

SW1990
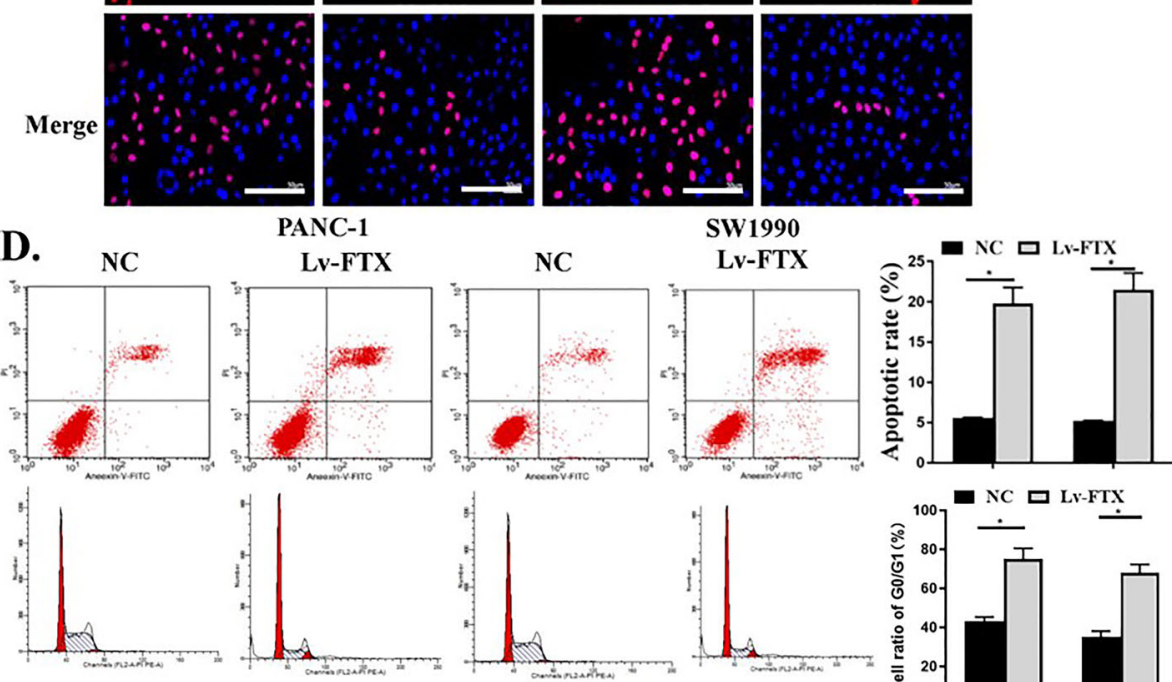

E.
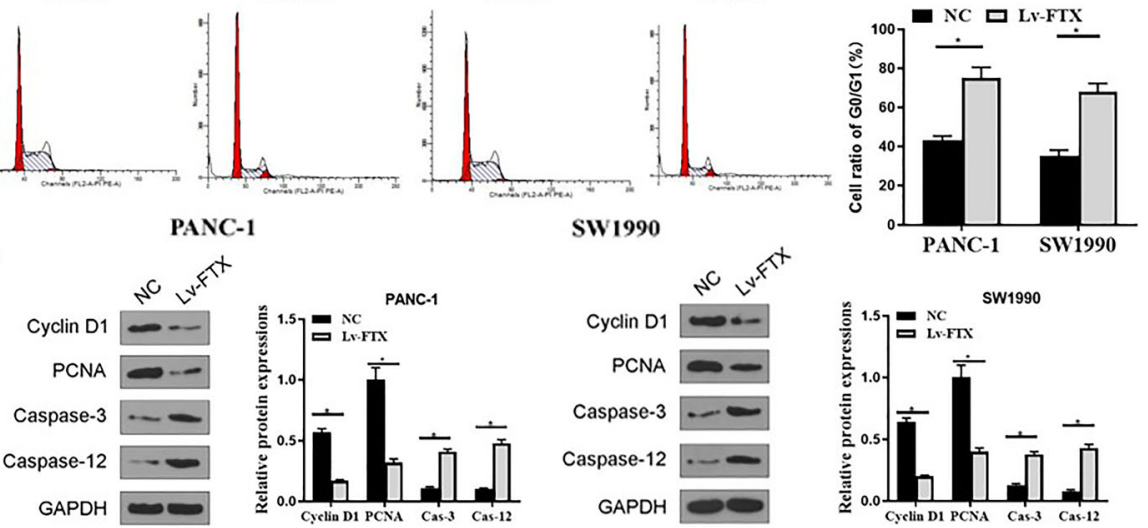

Fig. 2 Effects of silencing of FTX on proliferation and apoptosis of PC cells. a Measurement of the expression of FTX in PANC-1 and SW1990 cells by qRT-PCR. b, c Measurement of the proliferation activity of PANC-1 and SW1990 cells transfected with LV-FTX by CCK8 (b) and EDU (c) assays with EdU (red) and Hoechst 33342 (blue), compared with the control group. d Measurement of the apoptosis rates and cell cycle of PANC-1 and SW1990 cells between LV-FTX group and control group by flow cytometry. e Measurement of the protein expression of Cyclin D1, PCNA, cleaved caspase-3 (ccaspase-3) and cleaved caspase-12(c-caspase-12) in PANC-1 and SW1990 cells of LV-FTX and the control group by Western Blot. ${ }^{*} P<0.05$

the pathogenesis of PC by inhibiting the migration and invasion of PC cells.

\section{Identification of miR-513b-5p as the target of FTX}

It is known that lncRNAs can interact with miRNAs and act as competing endogenous RNAs [34]. Therefore, we speculated that FTX might interact with miRNAs in PC cells. Firstly, the potential binding site of FTX in miR513b-5p was predicted based on bioinformatics analysis using starBase v2.0 software (http://starbase.sysu.edu.cn/) (Fig. 4a). In addition, the transfected miR-513b-5p mimic observably increased the expression levels of miR-513b-5p 

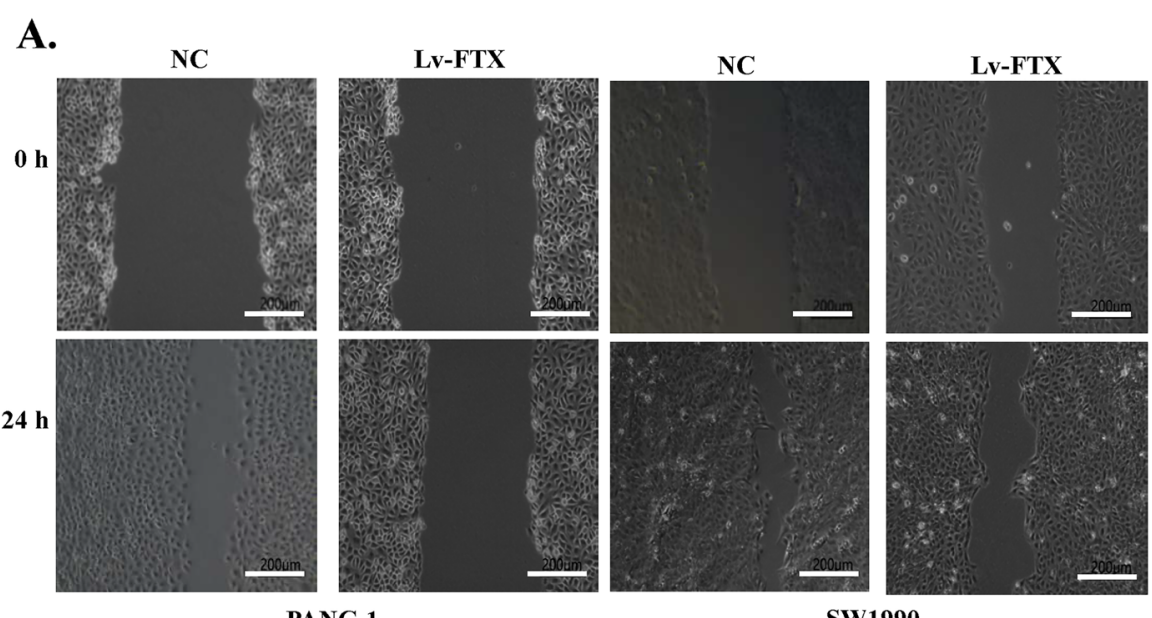

PANC-1

SW1990
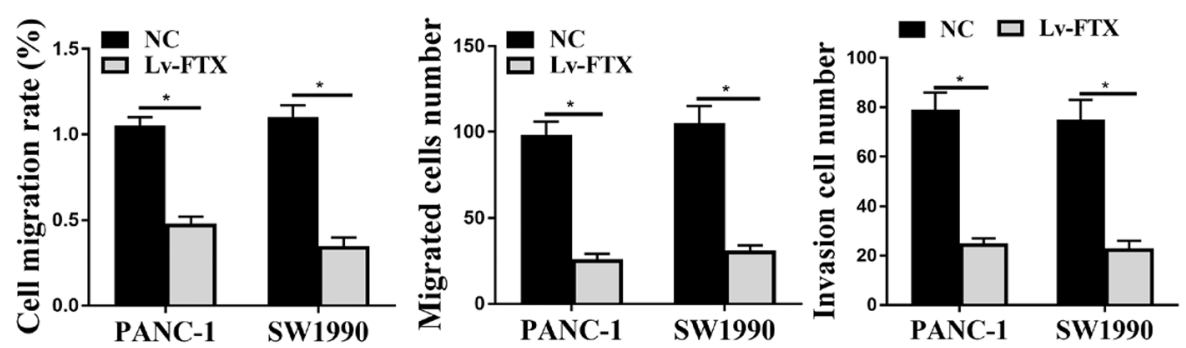

B. NC Lv-FTX

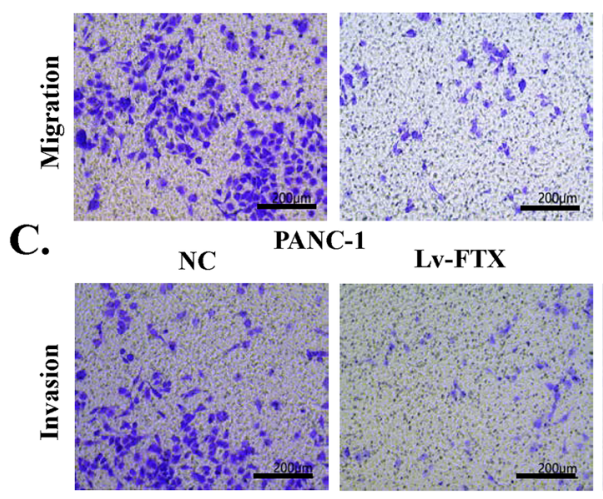

PANC-1 NC Lv-FTX

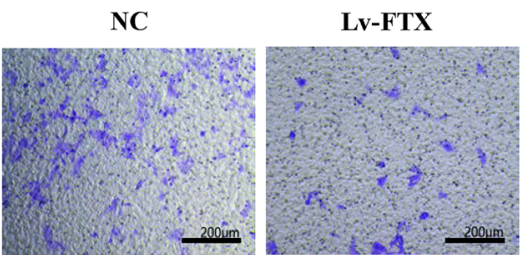

SW1990 Lv-FTX

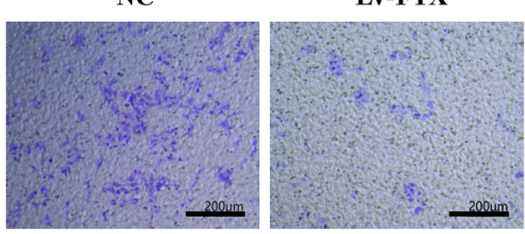

SW1990

Fig. 3 Effects of silencing of FTX on migration and invasion of PC cells. a Detection of the PANC-1 and SW1990 cells in LV-FTX and control group mobility by wound healing assay. b, c Detection of the ability of cell migration (b) and invasion (c) by Transwell assay. ${ }^{*} P<0.05$

(Fig. 4b, $P<0.05$ ), indicating that miR-513b-5p mimic was successfully transfected. Then, the direct binding between FTX and miR-513b-5p was verified by luciferin gene reporter assay. Compared to the control group, miR-513b$5 \mathrm{p}$ mimic significantly decreased the luciferase activity in FTX-WT cells (Fig. 4c, $P<0.05$ ), but had no significant effect on FTX-MUT cells $(P>0.05)$, indicating that FTX could bind with miR-513b-5p to reduce luciferase activity in FTX-WT cells. Meanwhile, RIP results further verified the direct binding between FTX and miR-513b-5p. As shown in Fig. 4d, compared to the control group, Bio-
miR-513 caused FTX to raise, indicating that mir-315 could bind to FTX. These results confirmed that miR513b-5p was a direct target of FTX.

\section{Effects of miR-513b-5p on PC cell proliferation and invasion}

To explore the effect of miR-513b-5p on cell behaviors, PANC-1 and SW1990 cells were treated with miR-513b$5 p$ inhibitor. Compared with the control group, miR513b-5p inhibitor observably inhibited the expression of miR-513b-5p in PANC-1 and SW1990 cells (Fig. 5a, $P<$ 

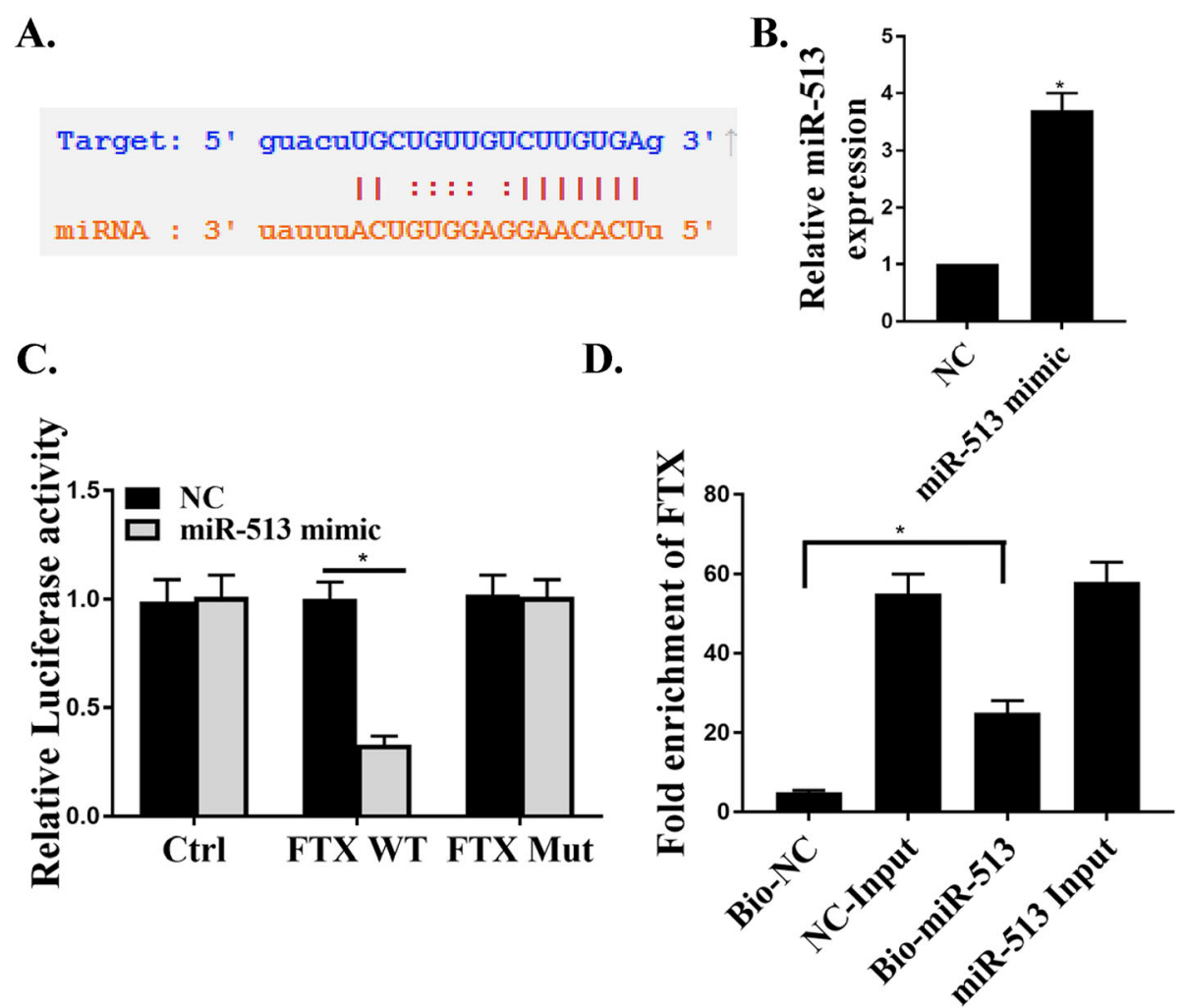

Fig. 4 Identification of miR-513b-5p as the target of FTX. a Schematic diagram of potential binding sites between miR-513-5p and FTX. b Determination of miR-513 expression in transfected miR-513b-5p mimic and negative control cells. c Detection of luciferase activity of transfected miR-513b-5p mimic group and negative control cells in control, FTX-WT or FTX-MUT cells. $\mathbf{d}$ Detection of FTX expression by RIP and qRT-PCR. * $P<0.05$

0.05), confirming that miR-513b-5p inhibitor could effectively suppress miR-513b-5p. In addition, the expression levels of miR-513b-5p in PANC-1 and SW1990 cells of LV-FTX groups were significantly higher than that in the control group (Fig. 5b, $P<0.05$ ), suggesting that silencing of FTX remarkably increased the expression levels of miR-513b-5p in PC cells. Furthermore, Edu assay showed that miR-513b-5p inhibitor observably increased the number of Edu-positive cells in PANC-1 and SW1990 cells (Fig. 5c and d, $P<0.05$ ), while silencing of FTX remarkably reversed this promotion $(P<$ 0.05). Moreover, Transwell assay showed that miR-513b$5 \mathrm{p}$ inhibitor remarkably promoted the invasion, migration (Fig. 5e and f, $P<0.05$ ) and invasion (Fig. $5 \mathrm{~g}$ and $\mathrm{h}$, $P<0.05)$ of PANC-1 and SW1990 cells, while silencing of FTX showed the opposite effect. These results demonstrated that silencing of FTX could effectively inhibit the proliferation and invasion of PC cells, whereas this phenomenon could be promoted by miR-513b-5p inhibitor in vitro.

\section{Effects of FTX on PC tumor growth in vivo}

The effects of FTX on PC tumor growth in vivo were investigated by establishing xenotransplantation mouse model. Mice were injected with transfected LV-NC or LV-FTX AsPC-1 cells that had a high transfection rate. Compared to the control group, PC tumor volume in LV-FTX group was remarkably decreased (Fig. 6a, $P<$ 0.05). In addition, compared to the control group, the expression of FTX in PC tumor tissues of LV-FTX group was observably down-regulated (Fig. 6b, $P<0.05$ ), indicating that LV-FTX successfully silenced FTX in PC tumor tissues. In addition, IHC results showed that, compared to the control group, the number of Ki-67 positive cells in PC tumor tissues of LV-FTC group was markedly decreased (Fig. 6c), suggesting that silencing of FTX inhibited the growth of PC cells in vivo. Meanwhile, the expression levels of miR-513b-5p in PC tumor tissues of LV-FTX group was observably higher than that of the control group (Fig. $6 \mathrm{~d}, P<0.05$ ), suggesting that silencing of FTX promoted the expression of miR-513b$5 p$ in PC tumor tissues. Finally, Western Blot results showed that, compared to the control group, the expression of PCNA, c-caspase-3 and c-caspase-12 in PC tumor tissues in LV-FTX group were remarkably upregulated (Fig. 6e, $P<0.05$ ), indicating that silencing of FTX promoted the apoptosis of PC cells and decreased cell proliferation. These results demonstrated that 


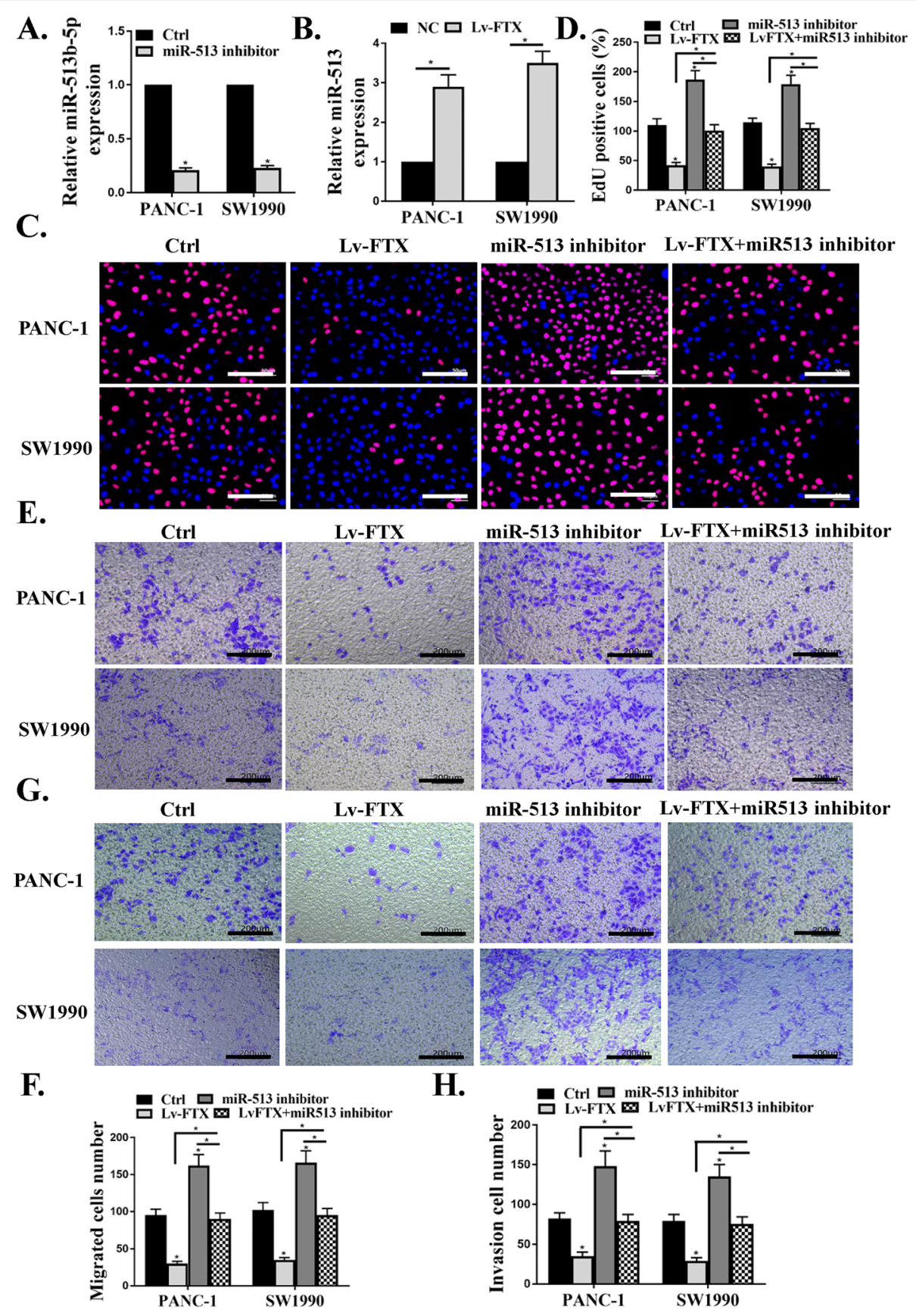

Fig. 5 Effects of miR-513b-5p on PC cell proliferation and invasion. a Effect of miR-513b-5p inhibitor on the expression of miR-513b-5p in PANC-1 and SW1990 cells. $\mathbf{b}$ Effect of silencing of FTX on the expression of miR-513b-5p in PANC-1 and SW1990 cells. c, $\mathbf{d}$ Measurement of the proliferation activity of PANC-1 and SW1990 cells by Edu assay with EdU (red) and Hoechst 33342 (blue). e, $\mathbf{f}$ Determination of the migration ability of PANC-1 and SW1990 cells. (G, H) Determination of the invasion ability of PANC-1 and SW1990 cells. ${ }^{*} P<0.05$

silencing of FTX could suppress the growth of PC tumors in xenotransplantation model mice.

\section{Discussion}

PC affects the digestive system and is one of the malignant tumors [35]. Extensive studies have identified a variety of IncRNAs that play important regulatory roles in the progression of PC [36, 37]. Abnormal expression of lncRNA FTX has been observed in liver cell carcinoma, renal cell carcinoma, colorectal cancer, and other cancers [23, 38]. However, the mechanism of FTX in PC is still unclear.

In this study, the expression of FTX in PC cell lines was determined by qRT-PCR, and it showed that FTX 
A.

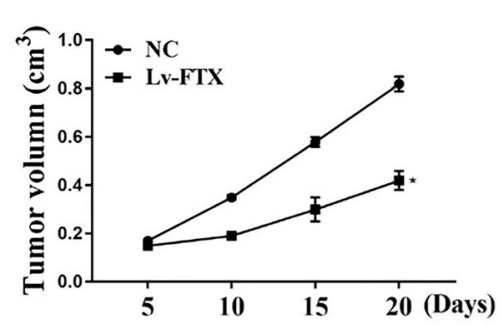

C.

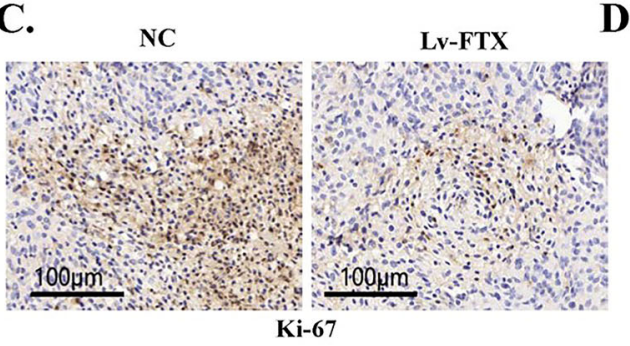

B.

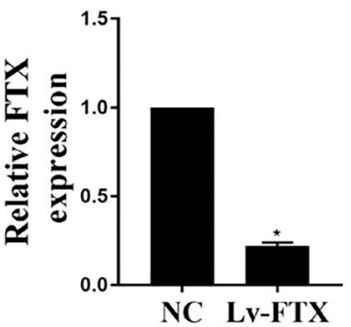

D.

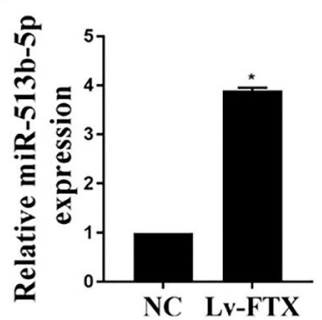

E.
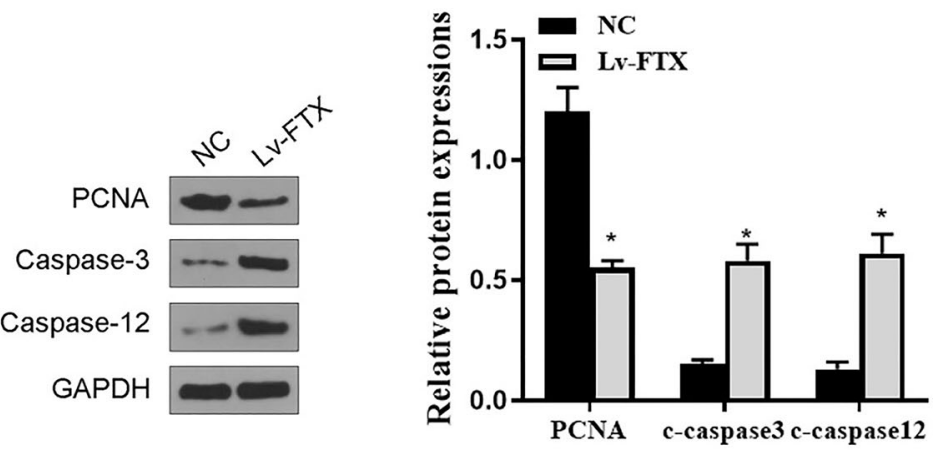

Fig. 6 Effects of FTX and miR-513b-5p on PC tumor growth in vivo. a Measurement of PC tumor volume in LV-FTX and control group of mice. $\mathbf{b}$ Determination of the expression of FTX in PC tumors in group of mice. c Ki-67 IHC staining in PC tumor tissues of mice. $\mathbf{d}$ Determination of the expression of miR-513b-5p in PC tumors in mice of group. (E) Determination of the expression levels of PCNA, c-caspase-3 and c-caspase- 12 in PC tumor tissues of group by Western Blot. $n=6,{ }^{*} P<0.05$

was up-regulated in all 5 PC cell lines, suggesting that FTX might be related to the development of PC. In addition, the expression levels of FTX were the highest in PANC-1 and SW1990 cells, so PANC-1 and SW1990 cells were selected for our follow-up in vitro experiments.

Malignant proliferation of cancer cells is one of the biological characteristics of cancer, and the imbalance of apoptosis is an important factor in the development of tumor $[39,40]$. Apoptosis is mainly regulated by death receptor mediated apoptosis pathway, mitochondrial pathway and endoplasmic reticulum pathway [41]. Caspase- 3 is the key executing enzyme in these pathways. After receiving the stimulation signal, the cells undergo a series of cascade reactions to activate procaspase-3, cleaved to the active caspase-3, and finally induce apoptosis [42]. Caspase-12 is involved in the regulation of activation pathways in ER stress [43]. In this study, we found that silencing of FTX remarkably inhibited cell activity and Edu positive cell number of PANC-1 and SW1990 cells, and increased the apoptosis rates and the expression levels of c-caspase-3 and ccaspase-12, confirming that silencing of FTX observably inhibited the proliferation activity of PC cells and promoted the apoptosis of cells. Invasion and metastasis are important biological characteristics of $\mathrm{PC}$, and advanced PC has the tendency of high distant metastasis and local invasion [44, 45]. We also observed that silencing of FTX induced PC cell cycle arrest at G0/G1 phase and decreased the expression levels of Cyclin D1 and PCNA. Silencing of FTX markedly suppressed the invasion and migration of SW1990 and PANC-1 cells, suggesting that silencing of FTX accelerated relieving of the symptoms of PC by inhibiting the invasion and migration of PC cells.

LncRNAs also play roles in the development of PC as competitive endogenous RNAs (ceRNAs) [46, 47]. CeRNA is a special manifestation of IncRNA, which can 
competitively combine with corresponding miRNAs, inhibit the activity of miRNAs, regulate the expression of downstream targeted genes, and thus regulating intracellular pathophysiological changes [48]. Studies have found that lncRNA PVT1 and miR-488 can promote the growth and invasion of PC by regulating ceRNA [36]. In addition, it was reported that FTX was upregulated in gliomas cells and could inhibit the expression of miR342-3p [49]. One study found that Ftx/miR-545 promoted proliferation and cell cycle progression of HCC cells through activation of the PI3K/Akt pathway [24]. However, another study showed the opposite results and found that FTX could inhibit HCC cell proliferation and activity by binding to MCM2 and miR-374a [50]. In this study, we found that the expression levels of miR-513b$5 p$ were decreased in PC cell lines, and inhibition of miR-513b-5p could remarkably promote the growth and invasion ability of PC cells, suggesting that miR-513b-5p might be related to the development of PC. Meanwhile, we found that miR-513b-5p was the direct target of FTX. Besides, by detecting malignant biological behaviors of PC cells, we showed that FTX could promote the growth, invasion and migration of PC cells by targeting miR-513b-5p.

Xenotransplantation model is an ideal animal model for tumor research [51, 52]. At present, xenografted mouse models have been established for PC, gastric cancer, breast cancer and other tumors, which maintained the pathological and histological characteristics of the original tumor from multiple perspectives such as histopathology and genetics [53-56]. In this study, the effects of FTX on PC tumor growth in vivo were investigated by establishing xenotransplantation mouse model. The results showed that silencing of FTX observably suppressed the growth of PC cells in mice. Meanwhile, silencing of FTX promoted the expression levels of miR513b-5p in PC tumor tissues, and up-regulated the expression of c-caspase- 3 and c-caspase-12 in PC tumor tissues, suggesting that silencing of FTX induces the apoptosis of PC cells in vivo. Combining in vivo and in vitro results, we confirmed that silencing of FTX suppressed pancreatic cancer cell proliferation and invasion by upregulating miR-513b-5p.

\section{Conclusion}

In summary, FTX was upregulated in PC cell lines and could inhibit the apoptosis of PC cells and promote their proliferation, migration and invasion by up-regulating miR-513b-5p. Therefore, silencing of FTX suppressed pancreatic cancer cell proliferation and invasion though upregulating miR-513b-5p. Identifying of the function of FTX could provide a new target and approach for the targeted therapy of PC.

\section{Abbreviations}

IncRNA: Long non-coding RNA; PC: Pancreatic cancer; qRT-PCR: Quantitative real-time PCR; RIP: RNA immunoprecipitation; HOTTIP: HOXA terminal transcriptional antisense RNA; miRNA: MicroRNA; IHC: Immuno-histochemical staining; ceRNA: Competitive endogenous RNA

\section{Supplementary Information}

The online version contains supplementary material available at https://doi. org/10.1186/s12885-021-07975-6.

Additional file 1: Supplementary figure-1. Original blot images of western blot

\section{Acknowledgments}

Not applicable.

\section{Authors' contributions}

SL and QZ: manuscript writing, experiment studies, literature search and data analysis; WL: data analysis and statistical analysis. CBZ: research design, manuscript editing and project administration. All authors read and approved the final manuscript.

\section{Funding}

We thank the financial support from Heilongjiang Provincial Health and Family Planning Commission on Scientific Research Project. The funding bodies had no role in the design of the study; collection, analysis, and interpretation of data and writing of the manuscript.

\section{Availability of data and materials}

The datasets used and/or analyzed during the current study are available from the corresponding author on reasonable request.

\section{Declarations}

Ethics approval and consent to participate

All procedures performed in studies involving animals were conducted in accordance with the Cancer Hospital of Harbin Medical University guidelines for the care and use of experimental animals strictly, and was approved by the ethics committee of Cancer Hospital of Harbin Medical University.

Consent for publication

Not applicable.

\section{Competing interests}

The authors declare that they have no competing interests.

Received: 7 August 2020 Accepted: 25 February 2021

Published online: 18 March 2021

\section{References}

1. Bailey P, Chang DK, Nones K, Johns AL, Patch A-M, Gingras M-C, et al. Genomic analyses identify molecular subtypes of pancreatic cancer. Nature. 2016;531(7592):47.

2. Panni R, Herndon J, Knolhoff B, Hogg G, Gupta V, Fields R, et al. CD11b hyperactivation improves response to immunotherapy in pancreatic cancer. HPB. 2018;20:S536-S7.

3. Chien W, Ding L, Sun Q, Torres-Fernandez L, Tan S, Xiao J, et al. Selective inhibition of unfolded protein response induces apoptosis in pancreatic cancer cells. Oncotarget. 2014;5(13):4881-94.

4. Chien W, Sudo M, Ding L, Sun Q, Wuensche P, Lee K, et al. Functional genome-wide screening identifies targets and pathways sensitizing pancreatic Cancer cells to Dasatinib. J Cancer. 2018;9(24):4762-73.

5. Kamisawa T, Wood LD, Itoi T, Takaori K. Pancreatic cancer. Lancet. 2016; 388(10039):73-85.

6. Takaori K, Bassi C, Biankin A, Brunner TB, Cataldo I, Campbell F, et al. International Association of Pancreatology (IAP)/European pancreatic Club (EPC) consensus review of guidelines for the treatment of pancreatic cancer. Pancreatology. 2016;16(1):14-27. 
7. Zheng X, Carstens JL, Kim J, Scheible M, Kaye J, Sugimoto H, et al. Epithelialto-mesenchymal transition is dispensable for metastasis but induces chemoresistance in pancreatic cancer. Nature. 2015;527(7579):525.

8. Takano S, Reichert M, Bakir B, Das KK, Nishida T, Miyazaki M, et al. Prrx1 isoform switching regulates pancreatic cancer invasion and metastatic colonization. Genes Dev. 2016;30(2):233-47.

9. von Karstedt S, Conti A, Nobis M, Montinaro A, Hartwig T, Lemke J, et al. Cancer cell-autonomous TRAIL-R signaling promotes KRAS-driven cancer progression, invasion, and metastasis. Cancer Cell. 2015;27(4):561-73.

10. Krebs AM, Mitschke J, Losada ML, Schmalhofer O, Boerries M, Busch H, et al. The EMT-activator Zeb1 is a key factor for cell plasticity and promotes metastasis in pancreatic cancer. Nat Cell Biol. 2017;19(5):518.

11. Ito H, Tanaka S, Akiyama Y, Shimada S, Adikrisna R, Matsumura S, et al. Dominant expression of DCLK1 in human pancreatic cancer stem cells accelerates tumor invasion and metastasis. PLoS One. 2016;11(1):e0146564.

12. Vennin C, Chin VT, Warren SC, Lucas MC, Herrmann D, Magenau A, et al. Transient tissue priming via ROCK inhibition uncouples pancreatic cancer progression, sensitivity to chemotherapy, and metastasis. Sci Transl Med. 2017;9(384):eaai8504

13. Chari ST, Kelly K, Hollingsworth MA, Thayer SP, Ahlquist DA, Andersen DK, et al. Early detection of sporadic pancreatic cancer: summative review. Pancreas. 2015;44(5):693.

14. Cohen JD, Javed AA, Thoburn C, Wong F, Tie J, Gibbs P, et al. Combined circulating tumor DNA and protein biomarker-based liquid biopsy for the earlier detection of pancreatic cancers. Proc Natl Acad Sci. 2017;114(38): 10202-7.

15. Pandya G, Kirtonia A, Sethi G, Pandey A, Garg M. The implication of long non-coding RNAs in the diagnosis, pathogenesis and drug resistance of pancreatic ductal adenocarcinoma and their possible therapeutic potential. Biochim Biophys Acta Rev Cancer. 1874;2020(2):188423.

16. Quinn JJ, Chang HY. Unique features of long non-coding RNA biogenesis and function. Nat Rev Genet. 2016;17(1):47.

17. Engreitz JM, Ollikainen N, Guttman M. Long non-coding RNAs: spatial amplifiers that control nuclear structure and gene expression. Nat Rev Mol Cell Biol. 2016;17(12):756.

18. Hajjari M, Salavaty A. HOTAIR: an oncogenic long non-coding RNA in different cancers. Cancer Biol Med. 2015;12(1):1.

19. Pang E-J, Yang R. Fu X-b, Liu Y-f. overexpression of long non-coding RNA MALAT1 is correlated with clinical progression and unfavorable prognosis in pancreatic cancer. Tumor Biol. 2015;36(4):2403-7.

20. Li Z, Zhao X, Zhou Y, Liu Y, Zhou Q, Ye H, et al. The long non-coding RNA HOTTIP promotes progression and gemcitabine resistance by regulating HOXA13 in pancreatic cancer. J Transl Med. 2015;13(1):84.

21. Cheng Y, Jutooru I, Chadalapaka G, Corton JC, Safe S. The long non-coding RNA HOTTIP enhances pancreatic cancer cell proliferation, survival and migration. Oncotarget. 2015;6(13):10840

22. Pan Y, Li C, Chen J, Zhang K, Chu X, Wang R, et al. The emerging roles of long noncoding RNA ROR (lincRNA-ROR) and its possible mechanisms in human cancers. Cell Physiol Biochem. 2016;40(1-2):219-29.

23. Yang Y, Zhang J, Chen X, Xu X, Cao G, Li H, et al. LncRNA FTX sponges miR215 and inhibits phosphorylation of vimentin for promoting colorectal cancer progression. Gene Ther. 2018;25(5):321-30.

24. Liu Z, Dou C, Yao B, Xu M, Ding L, Wang Y, et al. Ftx non coding RNAderived miR-545 promotes cell proliferation by targeting RIG-I in hepatocellular carcinoma. Oncotarget. 2016;7(18):25350-65.

25. He X, Sun F, Guo F, Wang K, Gao Y, Feng Y, et al. Knockdown of Long noncoding RNA FTX inhibits proliferation, migration, and invasion in renal cell carcinoma cells. Oncol Res. 2017;25(2):157-66.

26. Reddy KB. MicroRNA (miRNA) in cancer. Cancer Cell Int. 2015;15(1):38.

27. Yonemori K, Kurahara H, Maemura K, Natsugoe S. MicroRNA in pancreatic cancer. J Hum Genet. 2017;62(1):33.

28. Muti P, Donzelli S, Sacconi A, Hossain A, Ganci F, Frixa T, et al. MiRNA-513a$5 p$ inhibits progesterone receptor expression and constitutes a risk factor for breast Cancer: the ORDET prospective study. Carcinogenesis. 2017.

29. Higuchi T, Todaka H, Sugiyama Y, Ono M, Tamaki N, Hatano E, et al. Suppression of miR-7 biogenesis by NF90-NF45 controls cell proliferation in hepatocellular carcinoma. J Biol Chem. 2016;M116:748210.

30. Hu Y, Qin X, Yan D, Cao H, Zhou L, Fan F, et al. Genome-wide profiling of micro-RNA expression in gefitinib-resistant human lung adenocarcinoma using microarray for the identification of miR-149-5p modulation. Tumor Biol. 2017;39(3):1010428317691659.
31. Crossland RE, Norden J, Bibby LA, Davis J, Dickinson AM. Evaluation of optimal extracellular vesicle small RNA isolation and qRT-PCR normalisation for serum and urine. J Immunol Methods. 2016;429:39-49.

32. Guo P, Li N, Fan L, Lu J, Liu B, Zhang B, et al. Study of penetration mechanism of labrasol on rabbit cornea by Ussing chamber, RT-PCR assay, Western blot and immunohistochemistry. Asian J Pharm Sci. 2018.

33. Nakagawa T, Zhu H, Morishima N, Li E, Xu J, Yankner BA, et al. Caspase-12 mediates endoplasmic-reticulum-specific apoptosis and cytotoxicity by amyloid-beta. Nature. 2000;403(6765):98-103.

34. Cheng AM, Byrom MW, Shelton J, Ford LP. Antisense inhibition of human miRNAs and indications for an involvement of miRNA in cell growth and apoptosis. Nucleic Acids Res. 2005;33(4):1290-7.

35. Chittiboyina S, Chen Z, Chiorean EG, Kamendulis LM, Hocevar BA. The role of the folate pathway in pancreatic cancer risk. PLoS One. 2018;13(2): e0193298.

36. Zhao L, Kong H, Sun H, Chen Z, Chen B, Zhou M. LncRNA-PVT1 promotes pancreatic cancer cells proliferation and migration through acting as a molecular sponge to regulate miR-448. J Cell Physiol. 2018;233(5):4044-55.

37. Zhou Y, Chen Y, Ding W, Hua Z, Wang L, Zhu Y, et al. LncRNA UCA1 impacts cell proliferation, invasion, and migration of pancreatic cancer through regulating miR-96/FOXO3. IUBMB Life. 2018;70(4):276-90.

38. Li X, Zhao Q, Qi J, Wang W, Zhang D, Li Z, et al. IncRNA Ftx promotes aerobic glycolysis and tumor progression through the PPARgamma pathway in hepatocellular carcinoma. Int J Oncol. 2018;53(2):551-66.

39. Sadeghi Ekbatan S, Li X-Q, Ghorbani M, Azadi B, Kubow S. Chlorogenic acid and its microbial metabolites exert anti-proliferative effects, S-phase cellcycle arrest and apoptosis in human colon cancer caco-2 cells. Int J Mol Sci. 2018;19(3):723.

40. Khanam R, Kumar R, Hejazi II, Shahabuddin S, Meena R, Jayant V, et al. Piperazine clubbed with 2-azetidinone derivatives suppresses proliferation, migration and induces apoptosis in human cervical cancer HeLa cells through oxidative stress mediated intrinsic mitochondrial pathway. Apoptosis. 2018;23(2):113-31

41. Ma Z-J, Lu L, Yang J-J, Wang X-X, Su G, Wang Z-I, et al. Lariciresinol induces apoptosis in HepG2 cells via mitochondrial-mediated apoptosis pathway. Eur J Pharmacol. 2018;821:1-10.

42. Wang S, Long S, Xiao S, Wu W, Hann SS. Decoction of Chinese herbal medicine Fuzheng Kang-Ai induces lung cancer cell apoptosis via STAT3/ Bcl-2/caspase-3 pathway. Evid Based Complement Alternat Med. 2018;2018.

43. Salvamoser R, Brinkmann K, O'Reilly LA, Whitehead L, Strasser A, Herold MJ. Characterisation of mice lacking the inflammatory caspases-1/11/12 reveals no contribution of caspase-12 to cell death and sepsis. Cell Death Differ. 2019;26(6):1124-37.

44. Mao L, Le S, Jin X, Liu G, Chen J, Hu J. CSN5 promotes the invasion and metastasis of pancreatic cancer by stabilization of FOXM1. Exp Cell Res. 2019;374(2):274-81.

45. Singh SK, Mishra MK, Eltoum I-EA, Bae S, Lillard JW, Singh R. CCR5/CCL5 axis interaction promotes migratory and invasiveness of pancreatic cancer cells. Sci Rep. 2018;8(1):1323.

46. Ma M, Cai B, Jiang L, Abdalla BA, Li Z, Nie Q, et al. IncRNA-Six1 Is a Target of miR-1611 that Functions as a ceRNA to Regulate Six 1 Protein Expression and Fiber Type Switching in Chicken Myogenesis. Cells. 2018;7(12):243.

47. Wei DM, Jiang MT, Lin P, Yang H, Dang YW, Yu Q, et al. Potential ceRNA networks involved in autophagy suppression of pancreatic cancer caused by chloroquine diphosphate: a study based on differentially-expressed circRNAs, IncRNAs, miRNAs and mRNAs. Int J Oncol. 2019;54(2):600-26.

48. Zhou J, Liu M, Chen Y, Xu S, Guo Y, Zhao L. Cucurbitacin B suppresses proliferation of pancreatic cancer cells by ceRNA: effect of miR-146b-5p and InCRNA-AFAP1-AS1. J Cell Physiol. 2019;234(4):4655-67.

49. Zhang W, Bi Y, Li J, Peng F, Li H, Li C, et al. Long noncoding RNA FTX is upregulated in gliomas and promotes proliferation and invasion of glioma cells by negatively regulating miR-342-3p. Lab Investig. 2017;97(4):447-57.

50. Liu F, Yuan JH, Huang JF, Yang F, Wang TT, Ma JZ, et al. Long noncoding RNA FTX inhibits hepatocellular carcinoma proliferation and metastasis by binding MCM2 and miR-374a. Oncogene. 2016;35(41):5422-34.

51. Das Gupta A, Chen K-H, Wu D, Hoskin V, Mewburn J, Lima P, et al. Mitochondrial Drp1 Binding Partners MiD49 and MiD51 Are Epigenetically Upregulated in Lung Cancer: A Novel Mitochondrial-Targeted Approach to Cell Cycle Regulation and Cancer Therapy. In: B68 ONCOGENIC MUTATIONS, METASTASIS, AND NOVEL THERAPEUTICS: American Thoracic Society; 2019. p. A3952-A. 
52. Boettcher AN, Kiupel M, Adur M, Cocco E, Santin A, Charley S, et al. Abstract LB-042: successful tumor formation following xenotransplantation of primary human ovarian cancer cells into severe combined immunodeficient (SCID) pigs. AACR; 2018.

53. Draz H, Goldberg AA, Tomlinson Guns ES, Fazli L, Safe S, Sanderson JT. Autophagy inhibition improves the chemotherapeutic efficacy of cruciferous vegetable-derived diindolymethane in a murine prostate cancer xenograft model. Investig New Drugs. 2018;36(4):718-25.

54. Tureci M-KR, Woll S, Yamada T, Sahin U. Characterization of zolbetuximab in pancreatic cancer models. Oncoimmunology. 2019;8(1):e1523096.

55. Subhash W, Yeo MS, Wang L, Tan SH, Wong FY, Thuya WL, et al. Anti-tumor efficacy of Selinexor (KPT-330) in gastric cancer is dependent on nuclear accumulation of p53 tumor suppressor. Sci Rep. 2018;8(1):12248.

56. Fricke IB, De Souza R, Costa Ayub L, Francia G, Kerbel R, Jaffray DA, et al. Spatiotemporal assessment of spontaneous metastasis formation using multimodal in vivo imaging in HER2+ and triple negative metastatic breast cancer xenograft models in mice. PLoS One. 2018;13(5):e0196892.

\section{Publisher's Note}

Springer Nature remains neutral with regard to jurisdictional claims in published maps and institutional affiliations.

Ready to submit your research? Choose BMC and benefit from:

- fast, convenient online submission

- thorough peer review by experienced researchers in your field

- rapid publication on acceptance

- support for research data, including large and complex data types

- gold Open Access which fosters wider collaboration and increased citations

- maximum visibility for your research: over $100 \mathrm{M}$ website views per year

At $\mathrm{BMC}$, research is always in progress.

Learn more biomedcentral.com/submissions 